\title{
Specific distribution of lichens on Dodonaea viscosa L. in the restinga area of Itapuã State Park in Southern Brazil
}

\author{
Suzana M. de A. Martins ${ }^{1,3}$ and Marcelo P. Marcelli ${ }^{2}$
}

Received: 23.09.2010; accept: 18.08.2011

\begin{abstract}
Specific distribution of lichens on Dodonaea viscosa L. in the restinga area of Itapuã State Park in Southern Brazil). The results of the specific distribution of lichens sampled on 30 small Dodonaea viscosa L. trees located in a restinga area on the banks of Lagoa Negra in Itapuã State Park, Rio Grande do Sul, State are presented. The difference in species diversity among the habitats was proved through similarity tests. The twig was the habitat that has presented the greatest floristic differentiation and the branches were similar to the trunks. Lichen communities vary depending on habitat conditioned to the bark roughness, which changes according to the age of the substrate.
\end{abstract}

Key words: diversity, habitat, lichen community

RESUMO - (Distribuição específica de liquens sobre Dodonaea viscosa L. no Parque Estadual de Itapuã no sul do Brasil). São apresentados os resultados de distribuição específica de liquens amostrados em 30 arvoretas de Dodonaea viscosa L. localizadas em uma área de restinga às margens da lagoa Negra no Parque Estadual de Itapuã, Rio Grande do Sul. Através dos testes de similaridade foi comprovada a diferença na diversidade de espécies entre os hábitats. O ramo foi o hábitat que apresentou maior diferença e os galhos apresentaram similaridade com o tronco. A comunidade liquênica varia dependendo do hábitat e está condicionada à rugosidade da casca, que se modifica em função da idade do substrato.

Palavras-chave: comunidade liquênica, diversidade, hábitat

\section{Introduction}

Since preference for habitats and microhabitats is well-developed in lichens, small differences in chemical ( $\mathrm{pH}$ and mineral contents) and physical factors (light, temperature, humidity, wind, substrate porosity, toughness and roughness, etc.) can explain species replacement (Hale 1955, Brodo 1973).

Authors such as Werth (2001) and Cáceres et al. $(2007,2008)$ who studied factors that influence species composition of epiphytic lichens, have concluded that the most important is the macroclimatic gradient followed by the spatial variation and substrate variation. Several authors agree that the microclimate has a greater influence on establishing epiphytic communities than the substrate, since the phorophyte is a non continuous variable, unlike the environmental ecological variables that usually establish gradients (Cornelissen \& Gradstein 1990, Komposch \& Hafellner 2000, 2003, Cáceres et al. 2007, 2008).
Hawksworth (1975) stated that variations in the presence of corticolous lichens depends more on the physical nature of the bark than on tree species. Young trees with smooth bark usually present only crustose forms, many of them with a very thin thallus. When the tree begins to age and the bark becomes rougher, other lichen forms appear, such as crustose species with thicker thallus or large foliose species, as well as fruticose ones.

Factors such as tree age, exposure to sunlight and dust are of special importance for the kind of lichen community that will colonize tree trunks. Depending on the circumstances, this community may be poorer or richer than that on the twigs. Likewise, it may happen that, in more advanced stages, many bryophytes, especially mosses, form communities over wide areas, occupying the place of lichens (Degelius 1964, Hale 1983, Cornelissen \& Gradstein 1990, Wolf 1993).

\footnotetext{
1. Museu de Ciências Naturais da Fundação Zoobotânica do Rio Grande do Sul, Caixa Postal 1188, 90690-000 Porto Alegre, RS, Brazil 2. Instituto de Botânica, Caixa Postal 68041, 04045-972 São Paulo, SP, Brazil

3. Corresponding author: suzana-martins@fzb.rs.gov.br
} 
Pedersen (1980) analyzed epiphytic lichens in oak forests and found a homogeneous lichen community on the four sides in young trees, while on the older trees the community composition on the trunk sides was being modified. According to this author, these changes may be related to trunk roughness and microclimate.

The epiphytic community differs more strongly depending on trunk height, although a difference was also found in the trunk communities on trunks of different ages (Ruchty et al. 2001).

Thus, therefore the substrate structure and the physical environmental characteristics are among the principal factors affecting lichen distribution on trunks. The physical-chemical characteristics of tree bark, such as texture, hardness, water retention, $\mathrm{pH}$, macro and micro nutrient composition are essential for the establishment of the lichen community (Hale 1957, Pearson 1969, Brodo 1973, Jesberger \& Sheard 1973, Hawksworth \& Hill 1984, Marcelli 1996, Nash III 1996, Schmidt et al. 2001).

While knowledge on Brazilian tropical lichen ecology is rudimentary, a few quantitative studies have been published for South and Southeast region (Marcelli 1987, 1992, 1995, 1998, Käffer et al. 2007, 2009, 2010).

Brazilian restinga forests are formations rich in lichens species (Marcelli 1990, 1991), and there are few data on this subject. Therefore, knowing lichen diversity on Dodonaea viscosa L. in restinga areas of the Itapuã Park and verifying the lichen species distribution on it is a major contribution to lichen studies in these areas.

\section{Material and methods}

Study area - Itapuã State Park is located in the municipality of Viamão, at coordinates $50^{\circ} 50^{\prime}-51^{\circ}$ $05^{\prime} \mathrm{W}$ and $30^{\circ} 20^{\prime}-30^{\circ} 27^{\prime} \mathrm{S}, 57 \mathrm{~km}$ from the city of Porto Alegre and $60 \mathrm{~km}$ from the Atlantic Ocean. It has an area of 5,566 ha, with beaches, islands, lakes, fields, dunes, restinga forests, and granite hills.

The park forms an island of high biodiversity and shelters the fauna and flora of successive geological eras. The plant cover is much diversified, especially due to determinant environmental factors, including not only coastal restinga forests, but also granite hills, a very distinct formation in which generally forests and grasslands occur with a great variety of physiognomic-floristic types. On the top and the side of the mountains, the frequent rocky outcrops are covered by many lichen species and mosses. Around some rocky outcrops small tree-shrub groups occur and the vegetation is strongly influenced by the Atlantic Rainforest.

In the broader geomorphologic or phytogeographic sense, restinga forests in Rio Grande do Sul approximately match the physiographic region named "Costa" and the Coastal-Plain geomorphologic province (Fortes 1959, Delaney 1965). The vegetation in this region mostly covers wind deposits represented by fixed or movable dunes (Teixeira et al. 1986).

Field activities - Eleven four-day field trips were carried out: three excursions in May, June and December, 2003 , in order to get acquainted with the area, select the phorophyte and acquire previous knowledge of the lichen species. The expeditions to obtain quantitative data were carried out in April, 2004 and in January, July and August, 2005.

As lichens are not seasonal, it was not necessary to distribute the collections throughout the year. In order to verify sample sufficiency a collector's curve was made by relating the quantity of species sampled with the quantity of sampled phorophytes. At each phorophyte sampled new species were summed to the total. The collection work was finished after the analysis of 30 small trees, when the collector's curve became stabilized.

Phorophyte - The selected phorophyte species was Dodonaea viscosa (Sapindaceae), popularly known as red-broom ("vassoura-vermelha"). This species was chosen for its wide distribution in the area and because it presents high lichen diversity and cover.

Dodonaea viscosa may vary from shrubs to small trees 3 to 8 meters high. It is a low land species (5$300 \mathrm{~m}$ ) characteristic of restinga vegetation and banks near the coast and presents a wide and expressive dispersion along all of the Rio Grande do Sul State coast. It is a heliophyte and selective xerophyte species, very abundant, especially in restinga shrubby vegetation altered by man. In sandy soils, they usually form almost pure dense groupings (Reitz 1980).

Phorophyte standardization - Thirty individuals of D. viscosa were selected near Lagoa Negra place. The chosen shrubs were at least $2 \mathrm{~m}$ high with a $20 \mathrm{~cm}$ trunk circumference, for standardization.

The shrubs received a numbered identification plate and the precise positions were marked with a GPS. 
Phorophyte analysis - The sampling method used was an adaptation of the Rubber Method, which was devised to measure coverage and to count lichens around the trunk, collecting the coverage differences already in the field, since it is automatically adapted to several trunk circumferences (Marcelli 1987, 1992).

The intention of this study was to sample linear lichen distribution from the base of the trunk to the twig extremities. For this, the method was adapted by replacing the rubber by a tape measure, which does not stretch and performs the count and size measurements directly in millimeters or centimeters, resulting in an extremely precise notation of each specimen presented along the measured extension. This method was named "Tape Measure Method" and its use is described below.

Lichens were observed, measured and noted in the field, from the base of the trunk towards the top of the small trees, up to the limit height of $1.20 \mathrm{~m}$ to $1.50 \mathrm{~m}$. When the first branching or branch appeared, after the stipulated height, it was cut to be analyzed later in the laboratory, so that the sampling could reach the full length of all the twigs. The samples were standardized as much as possible, trying to collect twigs and/or branches at the same height; however there were cases of twigs that were more exposed to sun and dust, a variable that was impossible to control.

South, East, North and West oriented branches and twigs from every D. viscosa sampled were analyzed, as well as the same four sides of the trunks, determined by using a compass.

Species identification - For species identification, standard lichenological procedures were used, which consist in morphological and chemical analysis, spot test and ultraviolet light test (Canêz 2005, Jungbluth 2006).

Samples were identified at the laboratory of the Phanerogam Botany Section of the Museum of Natural Sciences at the Zoobotanical Foundation of Rio Grande do Sul. Specific literature was also consulted for the identification of genera in some major groups, such as Dodge (1964), Hale (1976), Arvidsson (1983), Jørgensen \& James (1983), Sérusiaux (1983), Galloway (1985), Swinscow \& Krog (1988), Moberg (1989), Scutari (1992, 1995), Elix (1994), Ribeiro (1998), Fleig (1997, 1999), Athi (2000), Eliasaro \& Adler (2000), Brodo et al. (2001), Eliasaro (2001), Galloway (2001), Sipman (2002) and others several taxonomics words cited on Marcelli \& Ahti (1998).

Data treatment - All data referring to each phorophyte were included in a Microsoft Excel sheet. This sheet has details of each lichen specimen analyzed, on trunk, branch and twig.

Twigs, branches and trunks, they were considered as different habitats.

Specific diversity per habitat - In order to recognize similarity patterns of the epiphytic community composition, the sample units were classified according to present species and their abundance transformed by the natural logarithm + constant $(\log x+1)$ through a cluster analysis, using Euclidean distance as similarity measure and the Minimum Variance as clustering method (Ward 1963).

In addition the specific diversities were compared for all the habitats through the Kruskal-Wallis analysis of variance test (ANOVA) $(\mathrm{H}=21.39, \mathrm{p}<0.005)$.

\section{Results}

The 10,887 specimens found on the 30 phorophyte were distributed in 126 species, of which 104 occurred on twigs, 89 on branches and 89 on trunks (table 1). The greatest percentage of these were foliose lichens, followed by crustose and fruticose forms. Trunks presented the greatest percentage of species with foliose habit $(79.16 \%)$ while species with crustose habit were concentrated on twigs $(97.43 \%)$ and those with fruticose habit, although few, were distributed throughout all three habitats (table 2, figure 1).

Table 3 shows that 16 species were restricted to twigs, $10(62.5 \%)$ represented by crustose lichens, five $(31.2 \%)$, by foliose and one $(6.7 \%)$ by fruticose. Nine species were restricted to trunks, seven $(77.8 \%)$ being foliose, one (11.1\%) fruticose and one (11.1\%) crustose. There were no species restricted to branches.

Twenty-one species contributed with more than 100 individuals each. All of them were more frequent on twigs, decreasing their number on branches, and consecutively on trunks; among these, Dirinaria picta, Lecanora subfusca, Cratiria americana, Parmotrema mesotropum and Lecanora cf. pallidofuscescens counted more than 500 individuals (table 4).

Based on the cluster analysis (figure 2) and Kruskal-Wallis, the twig habitat presented the greatest difference between trunks and branches; the branches have presented similarity with the trunk (table 5, figure 4).

In the lichen community on $D$. viscosa, although presenting the greater species diversity in every habitat, foliose lichens, were more numerous on trunks, while crustose lichens were more representative on twigs. 
Table 1. Total of species found on different habitats.

\begin{tabular}{|c|c|c|c|}
\hline Species & Twigs & Branches & Trunks \\
\hline Bacidia sp. & $\mathrm{X}$ & & $\mathrm{X}$ \\
\hline Buellia myriocarpa (D.C.) Mudd & $\mathrm{X}$ & $\mathrm{X}$ & \\
\hline Buellia polyspora (Willey) Vain. & $\mathrm{X}$ & $\mathrm{X}$ & $\mathrm{X}$ \\
\hline Bulbothrix goebelii (Zenker) Hale & $\mathrm{X}$ & $\mathrm{X}$ & $\mathrm{X}$ \\
\hline Bulbothrix isidiza (Nyl.) Hale & $\mathrm{X}$ & $\mathrm{X}$ & $\mathrm{X}$ \\
\hline Bulbothrix cf. semilunata (Lynge) Hale & $\mathrm{X}$ & & \\
\hline Bulbothrix tabacina (Mont. \& Bosch) Hale & $\mathrm{X}$ & $\mathrm{X}$ & $\mathrm{X}$ \\
\hline Bulbothrix ventricosa (Hale \& Kurok.) Hale & $\mathrm{X}$ & & $\mathrm{X}$ \\
\hline Bulbothrix sp. 1 & $\mathrm{X}$ & $\mathrm{X}$ & $\mathrm{X}$ \\
\hline Bulbothrix sp. 2 & & $\mathrm{X}$ & \\
\hline Candelaria sp. & & $\mathrm{X}$ & \\
\hline Candelariella sp. & $\mathrm{X}$ & & \\
\hline Canoparmelia carneopruinata (Zahlbr.) Elix \& Hale & $\mathrm{X}$ & $\mathrm{X}$ & $\mathrm{X}$ \\
\hline Canoparmelia caroliniana (Nyl.) Elix \& Hale & $\mathrm{X}$ & $\mathrm{X}$ & $\mathrm{X}$ \\
\hline Canoparmelia cinerascens (Lynge) Elix \& Hale & $\mathrm{X}$ & $\mathrm{X}$ & $\mathrm{X}$ \\
\hline Canoparmelia cf. conlabrosa Hale & $\mathrm{X}$ & $\mathrm{X}$ & \\
\hline Canoparmelia crozalsiana (de Lesd.) Elix \& Hale & $\mathrm{X}$ & $\mathrm{X}$ & $\mathrm{X}$ \\
\hline Canoparmelia cf. scrobicularis (Kremp.) Elix \& Hale & $\mathrm{X}$ & $\mathrm{X}$ & \\
\hline Canoparmelia texana (Tück.) Elix \& Hale & & $\mathrm{X}$ & $\mathrm{X}$ \\
\hline Canoparmelia sp. & $\mathrm{X}$ & & \\
\hline Coccocarpia erythroxyli (Spreng.) Sw. \& Krog & $\mathrm{X}$ & & $\mathrm{X}$ \\
\hline Coccocarpia palmicola (Spreng.) Arv. \& D.J. Galloway & $\mathrm{X}$ & $\mathrm{X}$ & $\mathrm{X}$ \\
\hline Coenogonium geralense (Henn.) Lücking & & & $\mathrm{X}$ \\
\hline Cratiria americana (Fée) Kalb \& Marbach & $\mathrm{X}$ & $\mathrm{X}$ & $\mathrm{X}$ \\
\hline Cryptothecia striata $\mathrm{G}$. Thor & & $\mathrm{X}$ & $\mathrm{X}$ \\
\hline Dirinaria applanata (Fée) Awasthi & $\mathrm{X}$ & $\mathrm{X}$ & $\mathrm{X}$ \\
\hline Dirinaria picta (Sw.) Clem. \& Shear & $\mathrm{X}$ & $\mathrm{X}$ & $\mathrm{X}$ \\
\hline Fissurina sp. & $\mathrm{X}$ & & \\
\hline Graphis adpressa Vain. & $\mathrm{X}$ & & \\
\hline Graphis anfractuosa Eschw. & $\mathrm{X}$ & $\mathrm{X}$ & \\
\hline Graphis assimilis Nyl. & $\mathrm{X}$ & $\mathrm{X}$ & \\
\hline Graphis librata Knight & $\mathrm{X}$ & $\mathrm{X}$ & $\mathrm{X}$ \\
\hline Graphis olivacea Redinger & $\mathrm{X}$ & $\mathrm{X}$ & $\mathrm{X}$ \\
\hline Graphis cf. tachygrapha Nyl. & $\mathrm{X}$ & $\mathrm{X}$ & \\
\hline Graphis sp. & $\mathrm{X}$ & & \\
\hline Gyrostomum scyphuliferum (Ach.) Nyl. & $\mathrm{X}$ & & $\mathrm{X}$ \\
\hline Haematomma sp. & $\mathrm{X}$ & $\mathrm{X}$ & $\mathrm{X}$ \\
\hline Heterodermia albicans (Pers.) Sw. \& Krog & & $\mathrm{X}$ & $\mathrm{X}$ \\
\hline Heterodermia diademata (Taylor) Awasthi & $\mathrm{X}$ & $\mathrm{X}$ & $\mathrm{X}$ \\
\hline Heterodermia obscurata (Nyl.) Trevis & & & $\mathrm{X}$ \\
\hline Heterodermia speciosa (Wulfen) Trevis & & & $\begin{array}{c}\mathrm{X} \\
\text { continue }\end{array}$ \\
\hline
\end{tabular}


Table 1 (continuation)

\begin{tabular}{|c|c|c|c|}
\hline Species & Twigs & Branches & Trunks \\
\hline Hyperphyscia adglutinata (Flörke) M. Mayrhofer \& Poelt & $\mathrm{X}$ & $\mathrm{X}$ & $\mathrm{X}$ \\
\hline Hyperphyscia syncolla (Tuck.) Kalb. & $\mathrm{X}$ & & $\mathrm{X}$ \\
\hline Hypotrachyna degelii (Hale) Hale & & $\mathrm{X}$ & $\mathrm{X}$ \\
\hline Hypotrachyna imbricatula (Zahlbr.) Hale & & & $\mathrm{X}$ \\
\hline Hypotrachyna intercalanda (Vain.) Hale & & $\mathrm{X}$ & $\mathrm{X}$ \\
\hline Hypotrachyna livida (Taylor) Hale & $\mathrm{X}$ & $\mathrm{X}$ & $\mathrm{X}$ \\
\hline Hypotrachyna palmarum (Lynge) Hale & & $\mathrm{X}$ & $\mathrm{X}$ \\
\hline Hypotrachyna pustulifera (Hale) Skorepa & & $\mathrm{X}$ & $\mathrm{X}$ \\
\hline Lecanactis sp. & $\mathrm{X}$ & $\mathrm{X}$ & \\
\hline Lecanora concilianda Vain. & $\mathrm{X}$ & $\mathrm{X}$ & $\mathrm{X}$ \\
\hline Lecanora frustulosa (Dickson) Schaer. & $\mathrm{X}$ & $\mathrm{X}$ & $\mathrm{X}$ \\
\hline Lecanora cf. macrescens Vain. & $\mathrm{X}$ & $\mathrm{X}$ & $\mathrm{X}$ \\
\hline Lecanora cf. pallidofuscescens Vain. & $\mathrm{X}$ & $\mathrm{X}$ & $\mathrm{X}$ \\
\hline Lecanora subfusca (L.) Ach. & $\mathrm{X}$ & $\mathrm{X}$ & $\mathrm{X}$ \\
\hline Lecanora sulphurescens Fée & $\mathrm{X}$ & $\mathrm{X}$ & $\mathrm{X}$ \\
\hline Lecidea canorubella $\mathrm{Nyl}$. & $\mathrm{X}$ & $\mathrm{X}$ & \\
\hline Lecidea cf. testaceoglauca Vain. & $\mathrm{X}$ & & \\
\hline Lecidea $\mathrm{sp}$. & $\mathrm{X}$ & & \\
\hline Leptogium sp. & & & $\mathrm{X}$ \\
\hline Maronea sp. & $\mathrm{X}$ & & \\
\hline Melaspilea $\mathrm{sp}$. & $\mathrm{X}$ & $\mathrm{X}$ & \\
\hline Micarea sp. & $\mathrm{X}$ & $\mathrm{X}$ & \\
\hline Myelochroa lindmanii (Lynge) Elix \& Hale & $\mathrm{X}$ & $\mathrm{X}$ & \\
\hline Ochrolechia pallescens (L.) A. Massal. & $\mathrm{X}$ & $\mathrm{X}$ & $\mathrm{X}$ \\
\hline Parmelinopsis minarum (Vain.) Elix \& Hale & & & $\mathrm{X}$ \\
\hline Parmotrema austrosinense (Zahlbr.) Hale & $\mathrm{X}$ & $\mathrm{X}$ & $\mathrm{X}$ \\
\hline Parmotrema catarinae Hale & $\mathrm{X}$ & $\mathrm{X}$ & $\mathrm{X}$ \\
\hline Parmotrema cetratum (Ach.) Hale & $\mathrm{X}$ & $\mathrm{X}$ & $\mathrm{X}$ \\
\hline Parmotrema eciliatum (Nyl.) Hale & $\mathrm{X}$ & $\mathrm{X}$ & $\mathrm{X}$ \\
\hline Parmotrema cf. epicladum (Hale) Fleig & $\mathrm{X}$ & $\mathrm{X}$ & $\mathrm{X}$ \\
\hline Parmotrema homotomum (Nyl.) Hale & & $\mathrm{X}$ & $\mathrm{X}$ \\
\hline Parmotrema conferendum Hale & & $\mathrm{X}$ & $\mathrm{X}$ \\
\hline Parmotrema haitiense (Hale) Hale & $\mathrm{X}$ & & $\mathrm{X}$ \\
\hline $\begin{array}{l}\text { Parmotrema lobuliferum (C.H. Ribeiro \& Marcelli) O. Blanco, A. Crespo, Divakar, } \\
\text { Elix \& Lumbsch }\end{array}$ & $\mathrm{X}$ & $\mathrm{X}$ & \\
\hline Parmotrema pilosum (Stizenb.) Krog. \& Sw. & $\mathrm{X}$ & $\mathrm{X}$ & \\
\hline Parmotrema recipiendum (Nyl.) Hale & $\mathrm{X}$ & & $\mathrm{X}$ \\
\hline Parmotrema subcaperatum (Kremp.) Hale & $\mathrm{X}$ & & \\
\hline Parmotrema subsumptum (Nyl.) Hale & $\mathrm{X}$ & $\mathrm{X}$ & $\mathrm{X}$ \\
\hline Parmotrema uruguense (Kremp.) Hale & $\mathrm{X}$ & $\mathrm{X}$ & $\mathrm{X}$ \\
\hline Parmotrema sp. & $\mathrm{X}$ & & $\begin{array}{c}\mathrm{X} \\
\text { continue }\end{array}$ \\
\hline
\end{tabular}


Table 1 (continuation)

\begin{tabular}{|c|c|c|c|}
\hline Species & Twigs & Branches & Trunks \\
\hline Parmotrema macrocarpum (Pers.) Hale & $\mathrm{X}$ & $\mathrm{X}$ & $\mathrm{X}$ \\
\hline Parmotrema madilynae A. Fletcher & $\mathrm{X}$ & $\mathrm{X}$ & $\mathrm{X}$ \\
\hline Parmotrema melanothrix (Mont.) Hale & $\mathrm{X}$ & $\mathrm{X}$ & $\mathrm{X}$ \\
\hline Parmotrema mesotropum (Müll.Arg.) Hale & $\mathrm{X}$ & $\mathrm{X}$ & $\mathrm{X}$ \\
\hline Parmotrema praesorediosum (Nyl.) Hale & $\mathrm{X}$ & $\mathrm{X}$ & $\mathrm{X}$ \\
\hline Parmotrema reticulatum (Taylor) M. Choisy & & $\mathrm{X}$ & $\mathrm{X}$ \\
\hline Parmotrema simulans (Hale) Hale & & $\mathrm{X}$ & $\mathrm{X}$ \\
\hline Parmotrema spinibarbe (Kurok.) Fleig & $\mathrm{X}$ & $\mathrm{X}$ & $\mathrm{X}$ \\
\hline Parmotrema tinctorum (Nyl.) Hale & $\mathrm{X}$ & $\mathrm{X}$ & $\mathrm{X}$ \\
\hline Parmotrema vainioi (A. L. Smith) Hale & $\mathrm{X}$ & & $\mathrm{X}$ \\
\hline Parmotrema yodae (Kurok.) Hale & $\mathrm{X}$ & & \\
\hline Parmotrema sp. & & & $\mathrm{X}$ \\
\hline Pertusaria ostiolata Dibben & $\mathrm{X}$ & $\mathrm{X}$ & \\
\hline Pertusaria sp. & $\mathrm{X}$ & & \\
\hline Phaeographis lobata (Eschw.) Müll. Arg. & $\mathrm{X}$ & $\mathrm{X}$ & \\
\hline Phaeographis cf. subtigrina (Vain.) Zahlbr. & $\mathrm{X}$ & & \\
\hline Physcia aipolia (Humb.) Fürnr. & $\mathrm{X}$ & $\mathrm{X}$ & $\mathrm{X}$ \\
\hline Physcia alba (Fée) Müll. Arg. & $\mathrm{X}$ & $\mathrm{X}$ & $\mathrm{X}$ \\
\hline Physcia crispa Nyl. & $\mathrm{X}$ & $\mathrm{X}$ & $\mathrm{X}$ \\
\hline Physcia poncinsii Hue & $\mathrm{X}$ & $\mathrm{X}$ & \\
\hline Physcia stellaris (L.) Nyl. & $\mathrm{X}$ & $\mathrm{X}$ & \\
\hline Porina sp. & $\mathrm{X}$ & & \\
\hline Punctelia constantimontium Sérus. & $\mathrm{X}$ & & $\mathrm{X}$ \\
\hline Punctelia sp. & & & $\mathrm{X}$ \\
\hline Pyxine cocoës (Sw.) Nyl. & $\mathrm{X}$ & & \\
\hline Pyxine subcinerea Stirt. & $\mathrm{X}$ & & $\mathrm{X}$ \\
\hline Ramalina cf. calcarata Krog \& Sw. & $\mathrm{X}$ & $\mathrm{X}$ & \\
\hline Ramalina celastri (Spreng.) Krog \& Sw. & $\mathrm{X}$ & $\mathrm{X}$ & $\mathrm{X}$ \\
\hline Ramalina complanata (Sw.) Ach. & $\mathrm{X}$ & $\mathrm{X}$ & $\mathrm{X}$ \\
\hline Ramalina cf. exiguella Stirt. & $\mathrm{X}$ & $\mathrm{X}$ & $\mathrm{X}$ \\
\hline Ramalina grumosa Kashiwadani & $\mathrm{X}$ & $\mathrm{X}$ & $\mathrm{X}$ \\
\hline Ramalina peruviana Ach. & $\mathrm{X}$ & $\mathrm{X}$ & $\mathrm{X}$ \\
\hline Ramalina cf. sprengelii Krog \& Sw. & & & $\mathrm{X}$ \\
\hline Ramalina usnea (L.) Howe & $\mathrm{X}$ & $\mathrm{X}$ & $\mathrm{X}$ \\
\hline Ramalina sp. & $\mathrm{X}$ & & \\
\hline Ramboldia russula (Ach.) Kalb, Lumbsch \& Elix & $\mathrm{X}$ & $\mathrm{X}$ & $\mathrm{X}$ \\
\hline Rinodina sp. & $\mathrm{X}$ & $\mathrm{X}$ & $\mathrm{X}$ \\
\hline Teloschistes exilis (Michx.) Vain. & $\mathrm{X}$ & $\mathrm{X}$ & $\mathrm{X}$ \\
\hline Teloschistes flavicans (Sw.) Norman & $\mathrm{X}$ & $\mathrm{X}$ & $\mathrm{X}$ \\
\hline Usnea sp. 1 & $\mathrm{X}$ & $\mathrm{X}$ & $\mathrm{X}$ \\
\hline Usnea sp. 2 & $\mathrm{X}$ & $\mathrm{X}$ & $\begin{array}{c}\mathrm{X} \\
\text { continue }\end{array}$ \\
\hline
\end{tabular}


Table 1 (continuation)

\begin{tabular}{lccc}
\hline Species & Twigs & Branches & Trunks \\
\hline Usnea sp. 3 & $\mathrm{X}$ & $\mathrm{X}$ & $\mathrm{X}$ \\
Usnea sp. 4 & $\mathrm{X}$ & & $\mathrm{X}$ \\
Vainionora sp. & $\mathrm{X}$ & $\mathrm{X}$ & \\
Verrucaria sp. & $\mathrm{X}$ & $\mathrm{X}$ & $\mathrm{X}$ \\
\hline Total & 104 & 89 & 89 \\
\hline
\end{tabular}

Table 2. Total number of species and number of species (ns) per habit found on the different habitats.

\begin{tabular}{lccccccc}
\hline Habit & $\begin{array}{c}\text { Total number } \\
\text { of species }\end{array}$ & \multicolumn{2}{c}{ Twigs } & \multicolumn{2}{c}{ Branches } & \multicolumn{2}{c}{ Trunks } \\
\cline { 2 - 8 } & 72 & ns & $\%$ & ns & $\%$ & ns & $\%$ \\
\hline Foliose & 39 & 52 & 72 & 49 & 68 & 57 & 79 \\
Crustose & 38 & 97 & 27 & 69 & 19 & 49 \\
Fruticose & 14 & 14 & 87 & 13 & 81 & 13 & 81 \\
\hline
\end{tabular}

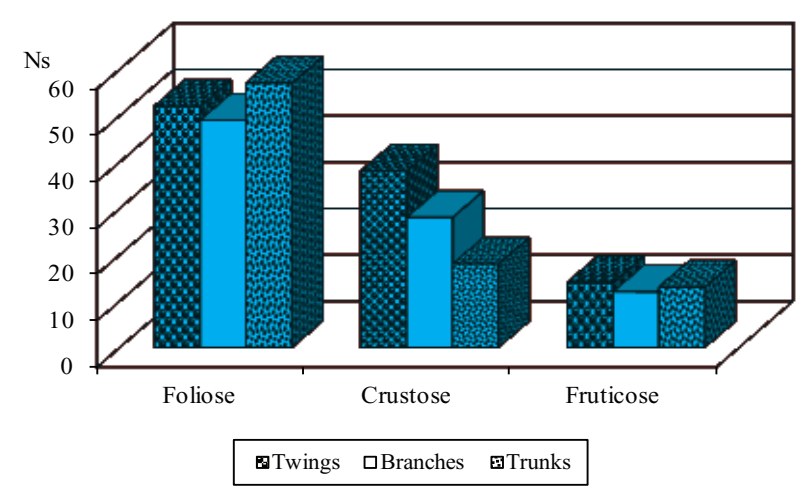

Figure 1. Graphic representation of the number of species per habit on the different habitats.

The most common foliose species found on the twigs were Dirinaria picta and Parmotrema mesotropum, which were fertile despite presenting small thalli. Their thalli sizes were larger on trunks, consequently increasing their coverage on that habitat.

Parmeliaceae occurred most among the foliose lichens, especially because of the Parmotrema species. Four species of this genus growed usually grouped: P. mesotropum, $P$. praesorediosum, . tinctorum and $P$. austrosinense.

Trunks - Eighteen lichen species presented preference for the trunks. Nine were exclusive and the other nine had their greater number of individuals on this habitat. Among these species, only two are not foliose: Ramalina cf. sprengelii (fruticose) and Coenogonium geralense (crustose).
The trunk is the habitat with the roughest bark and by consequence probably has more humidity, which allows the occurrence of species that are more demanding of this factor.

Degelius (1964) considered the species that are more or less rare on trunks as late immigrants; these are fruticose and foliose species that do not belong to the typical twig community. It is quite possible that the nine species that were exclusive to trunks are late immigrants in the community of D. viscosa (table 6).

Branches - A total of 89 species were found on the branches, 50 of them growing both on twigs and on trunks, 17 in common with twigs and only 21 with trunks (table 7). According to the statistical tests of specific diversity, this habit did not present significant differences when compared to the twigs, but showed more similarity with the trunks.

None of the species demonstrated preference for the branches.

Twigs - A total of 104 species occurred on the twigs. This is the habitat with the highest specific diversity and where species have presented the greatest number of individuals.

Some species appeared on the terminal portions of twigs and continued up to the insertion of the twig on the branch; other species have appeared less frequently on certain points.

Species that develop on such extremities are considered as settlers; however some differences 
Table 3. Species that have occurred only on twigs or trunks with their habit, where cr: crustose, fo: foliose and fr: fruticose.

\begin{tabular}{lllc}
\hline Twigs & Habit & Trunk & Habit \\
\hline Graphis adpressa & $\mathrm{cr}$ & Heterodermia speciosa & fo \\
Candelariella sp. & $\mathrm{cr}$ & Heterodermia obscurata & fo \\
Fissurina sp. & $\mathrm{cr}$ & Hypotrachyna imbricatula & fo \\
Graphis sp. & $\mathrm{cr}$ & Leptogium sp. & fo \\
Lecidea cf. testaceoglauca & $\mathrm{cr}$ & Parmelinopsis minarum & fo \\
Lecidea sp. & $\mathrm{cr}$ & Parmotrema sp. & fo \\
Maronea sp. & $\mathrm{cr}$ & Punctelia sp. & fo \\
Pertusaria sp. & $\mathrm{cr}$ & Ramalina cf. sprengelii & fr \\
Phaeographis cf. subtigrina & $\mathrm{cr}$ & Coenogonium geralense & $\mathrm{cr}$ \\
Porina sp. & $\mathrm{cr}$ & & \\
Parmotrema subcaperatum & fo & & \\
Canoparmelia sp. & fo & & \\
Bulbothrix cf. semilunata & fo & & \\
Parmotrema yodae & fo & & \\
Pyxine cocoës & fo & & \\
Ramalina sp. & fr & & \\
\hline
\end{tabular}

may occur among the twigs of a same tree. These variations depend on the exposure to sun and dust (Degelius 1964).

As a general rule, young trees and the twigs extremities present a smoother bark and, because of this, are more appropriate to the establishment of crustose forms, many of them with a very thin thallus. When the tree begins to get old and the bark become rougher, other species begin to appear, as crustose forms of thicker thallus or big folioses and fruticoses.

On the terminal twigs of $D$. viscosa a great number of individuals with very small thalli $(0.5 \mathrm{~cm}$ diameter) of Dirinaria picta occurred. Physcia crispa was also found, however in a smaller number.

Among the foliose species, Parmotrema mesotropum should be pointed out as the species of the genus with the greatest number of individuals on twigs. The individuals of $P$. mesotropum, even with a smaller thallus, were well developed, producing ascospores in the apothecia.

There were more foliose species than other groups in all habitats; however, the greatest numbers of individuals were of crustose forms, especially on the twigs.

Considering smooth surfaces, the crustose life forms start the succession. Although with a much reduced thallus, species such as Cratiria americana, Graphis assimilis, G. librata, Lecanora cf. pallidofuscescens and L. subfusca presented a very abundant production of ascomata. These species, together with Dirinaria picta (foliose) were the most numerous on twigs.

\section{Discussion}

The habitats presented a great difference between them, and the branches presented similarity to the twigs and trunks, being characterized as a transition zone. It may be explained by the ageing of substrate and this fact is directly related to bark roughness, since, as time goes by, the branches begin to present similar surface characteristics to the trunk, turning it into an ecotone transition zone, with species that occur on twigs as well as on trunks. Since it is a transition zone, it is the habitat where the species replacement in response to their different environment occurs. The branches, older than the twigs, present a rougher bark, more similar to trunks, which justifies the higher similarity between these habitats.

According to Hawksworth (1975), humidity caused by bark roughness may be an important factor in establishing different species that attach themselves more easily to irregular surfaces, but less so to smooth surfaces, and preference for a substrate depends more 
Table 4. Species that have contributed with more than 100 individuals, number of presence on the habitats and absolute frequency. Habits - cr: crustose, fo: foliose and fr: fruticose.

\begin{tabular}{lcrrrrr}
\hline & & \multicolumn{5}{c}{ Number of individuals } \\
\cline { 3 - 7 } \multicolumn{1}{c}{ Species } & Habit & Twigs & Branches & Trunks & Total & AF\% \\
\hline Dirinaria picta & fo & 1047 & 302 & 215 & 1564 & 100 \\
Lecanora subfusca & cr & 1134 & 183 & 91 & 1408 & 97 \\
Cratiria americana & cr & 583 & 90 & 23 & 696 & 90 \\
Parmotrema mesotropum & fo & 339 & 211 & 128 & 678 & 100 \\
Lecanora cf. pallidofuscescens & cr & 566 & 86 & 14 & 666 & 90 \\
Usnea sp. 2 & fr & 269 & 152 & 64 & 485 & 70 \\
Usnea sp. 3 & fr & 355 & 68 & 31 & 454 & 80 \\
Parmotrema praesorediosum & fo & 124 & 87 & 160 & 371 & 100 \\
Parmotrema tinctorum & fo & 156 & 54 & 153 & 363 & 100 \\
Ochrolechia pallescens & cr & 209 & 55 & 72 & 336 & 97 \\
Melaspilea sp. & cr & 307 & 3 & 0 & 310 & 97 \\
Parmotrema austrosinense & fo & 123 & 67 & 58 & 248 & 80 \\
Graphis assimilis & cr & 178 & 27 & 0 & 205 & 60 \\
Ramalina peruviana & fr & 130 & 25 & 26 & 181 & 73 \\
Ramalina celastri & fr & 99 & 57 & 21 & 177 & 67 \\
Graphis librata & cr & 158 & 14 & 2 & 174 & 73 \\
Bulbothrix goebelii & fo & 68 & 28 & 18 & 114 & 57 \\
Ramalina grumosa & fr & 93 & 30 & 2 & 125 & 57 \\
Parmotrema cetratum & fo & 11 & 18 & 84 & 113 & 70 \\
Hyperphyscia adglutinata & fo & 93 & 4 & 14 & 111 & 40 \\
Graphis olivacea & cr & 78 & 4 & 20 & 102 & 47 \\
\hline
\end{tabular}

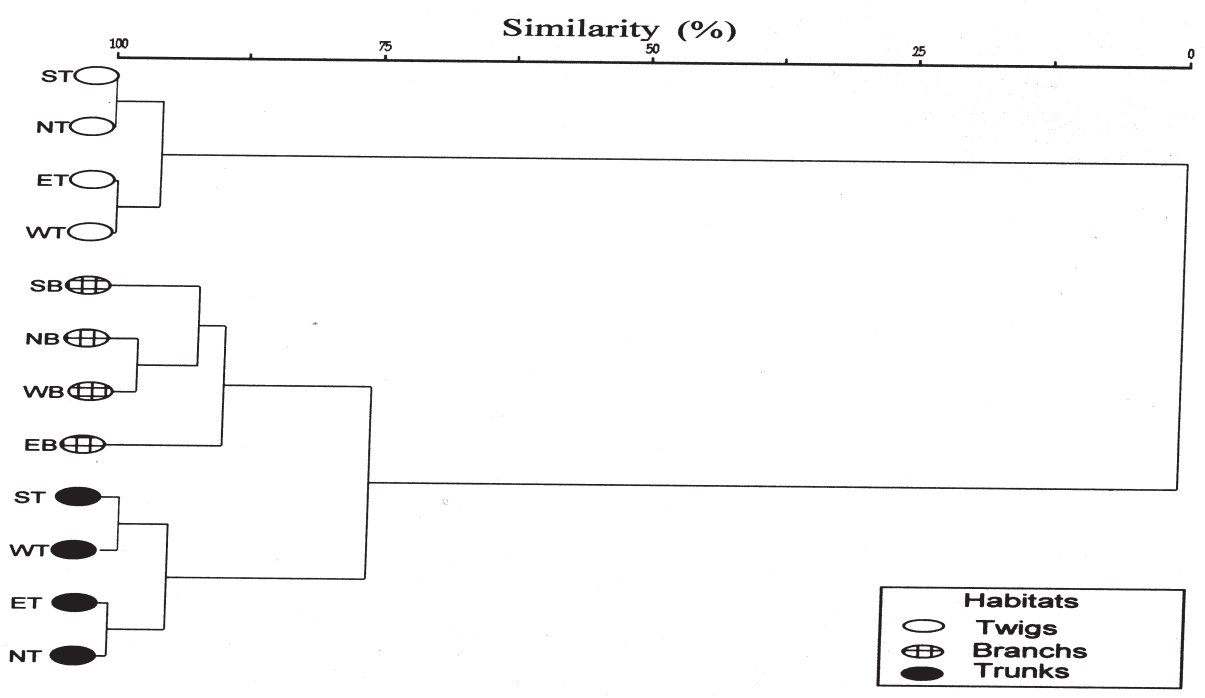

Figure 2. Similarity among habitats through the cluster analysis. where: ST: South Twig, NT: North Twig, WT: West Twig, ET: East Twig, SB: South Branch, NB: North Branch, WB: West branch, EB: East Branch, ST: South Trunk, NT: North Trunk, WT: West Trunk, ET: East Trunk. 
Table 5. Table of ANOVA for the relation between the species diversity and the habitats. Fonts of variations represent the habitats (base of the trunk, trunk and extremity of twigs). SS: Sum of squares, df: degrees of freedom, MS: Mean Squared, F: reason among the variances.

\begin{tabular}{lccccc}
\hline \multicolumn{6}{c}{ Species diversity } \\
\hline Font & $\mathrm{SS}$ & $\mathrm{df}$ & $\mathrm{MS}$ & $\mathrm{F}$ & $\mathrm{P}$ \\
\hline Habitats & 704.570 .173 & 2 & 352.285 .086 & 5.167 & 0.007 \\
Error & 1.04 & & 68.174 .423 & & \\
\hline
\end{tabular}

Table 6. Species that have presented preference for the trunk with the individual's number by habitat and their habit, where fo: foliose, fr: fruticose and cr: crustose.

\begin{tabular}{|c|c|c|c|c|}
\hline Species & Twig & Branch & Trunk & Habitat \\
\hline Parmotrema melanothrix & 28 & 2 & 43 & fo \\
\hline Parmotrema cetratum & 11 & 18 & 84 & fo \\
\hline Parmotrema madilynae & 9 & 13 & 26 & fo \\
\hline Coccocarpia palmicola & 6 & 9 & 53 & fo \\
\hline Canoparmelia caroliniana & 2 & 2 & 19 & fo \\
\hline Punctelia constantimontium & 1 & 0 & 20 & fo \\
\hline Hypotrachyna degelii & & 2 & 15 & fo \\
\hline Hypotrachyna pustulifera & & 2 & 10 & fo \\
\hline Parmotrema reticulatum & & 1 & 30 & fo \\
\hline Parmelinopsis minarum & & & 7 & fo \\
\hline Coenogonium geralense & & & 4 & $\mathrm{cr}$ \\
\hline Hypotrachyna imbricatula & & & 2 & fo \\
\hline Ramalina cf. sprengelii & & & 2 & $\mathrm{fr}$ \\
\hline Heterodermia speciosa & & & 1 & fo \\
\hline Heterodermia obscurata & & & 1 & fo \\
\hline Leptogium sp. & & & 1 & fo \\
\hline Parmotrema sp. & & & 1 & fo \\
\hline Punctelia sp. & & & 1 & fo \\
\hline
\end{tabular}

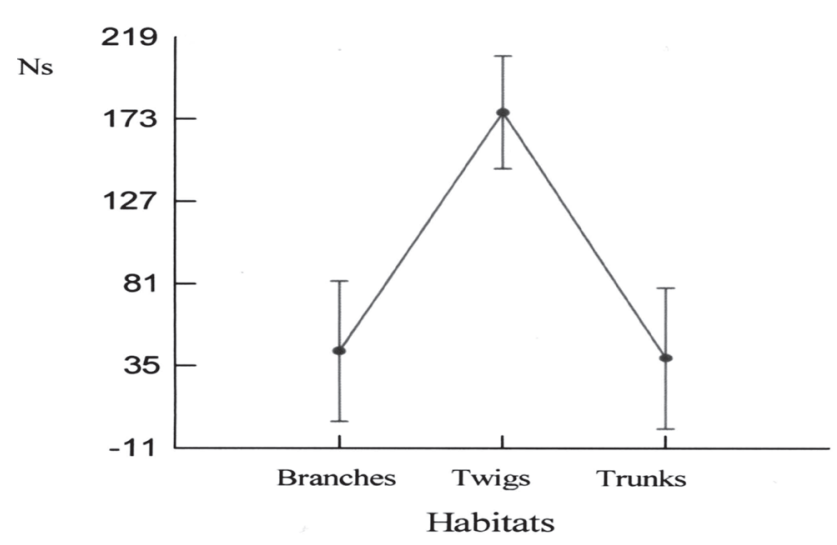

Figure 3. Similarity in species diversity among branches, twigs and trunks, through ANOVA. on the physical characteristics of the bark than on the phorophyte species. Trees with smooth bark and young trees in general present lichens of crustose habit; when the bark begins to become older and rougher, other species begin to appear.

According to Ruchty et al. (2001), with age and trunk development the process of colonization and competition begins, and there is species substitution changing all epiphytic life forms.

Hawksworth \& Hill (1984) have found a low density of crustose species on older twigs, attributed to their inability to compete with the foliose species, which grow faster and cover them.

The lichen community on $D$. viscosa was constant on the three habitats: twigs, branches and trunks. 
Table 7. Number of individuals of the 89 species found on the branches and their occurrence on twigs and trunks.

\begin{tabular}{|c|c|c|c|}
\hline Species & Branches & Twigs & Trunks \\
\hline Dirinaria picta & 302 & 1047 & 215 \\
\hline Parmotrema mesotropum & 211 & 339 & 128 \\
\hline Lecanora subfusca & 183 & 1134 & 91 \\
\hline Usnea sp. 2 & 152 & 269 & 64 \\
\hline Parmotrema minarum & 123 & 67 & 58 \\
\hline Cratiria americana & 90 & 583 & 23 \\
\hline Parmotrema praesorediosum & 87 & 124 & 160 \\
\hline Lecanora cf. pallidofuscescens & 86 & 566 & 14 \\
\hline Usnea sp. 3 & 68 & 355 & 31 \\
\hline Parmotrema austrosinense & 67 & 123 & 58 \\
\hline Ramalina celastri & 57 & 99 & 21 \\
\hline Ochrolechia pallescens & 55 & 209 & 72 \\
\hline Parmotrema tinctorum & 54 & 156 & 153 \\
\hline Ramalina usnea & 44 & 420 & 20 \\
\hline Ramalina grumosa & 30 & 93 & 2 \\
\hline Bulbothrix goebelii & 28 & 68 & 18 \\
\hline Lecanora sulphurescens & 26 & 49 & 2 \\
\hline Ramalina peruviana & 25 & 130 & 26 \\
\hline Pyrrhospora russula & 21 & 31 & 18 \\
\hline Haematomma sp. & 19 & 38 & 6 \\
\hline Parmotrema cetratum & 18 & 11 & 84 \\
\hline Ramalina complanata & 15 & 51 & 17 \\
\hline Graphis librata & 14 & 158 & 2 \\
\hline Parmotrema madilynae & 13 & 9 & 26 \\
\hline Lecanora $\mathrm{cf}$ macrescens & 10 & 17 & 3 \\
\hline Coccocarpia palmicola & 9 & 6 & 53 \\
\hline Physcia alba & 9 & 4 & 2 \\
\hline Parmotrema melanothrix & 7 & 28 & 43 \\
\hline Buellia polyspora & 7 & 14 & 1 \\
\hline Physcia aipolia & 6 & 13 & 2 \\
\hline Heterodermia diademata & 5 & 7 & 4 \\
\hline Hyperphyscia adglutinata & 4 & 93 & 14 \\
\hline Graphis olivacea & 4 & 78 & 20 \\
\hline Teloschistes flavicans & 4 & 19 & 7 \\
\hline Ramalina cf. exiguella & 4 & 15 & 4 \\
\hline Canoparmelia crozalsiana & 4 & 5 & 3 \\
\hline Parmotrema eciliatum & 4 & 3 & 5 \\
\hline Bulbothrix isidiza & 3 & 17 & 13 \\
\hline Dirinaria applanata & 3 & 17 & 2 \\
\hline Lecanora frustulosa & 3 & 10 & 13 \\
\hline Usnea sp. 1 & 3 & 9 & $\begin{array}{l}11 \\
\text { con }\end{array}$ \\
\hline
\end{tabular}


Table 7 (continuation)

\begin{tabular}{|c|c|c|c|}
\hline Species & Branches & Twings & Trunks \\
\hline Parmotrema uruguense & 3 & 1 & 2 \\
\hline Parmotrema conferendum & 3 & 4 & 6 \\
\hline Physcia crispa & 2 & 5 & 4 \\
\hline Canoparmelia cinerascens & 2 & 4 & 5 \\
\hline Canoparmelia caroliniana & 2 & 2 & 19 \\
\hline Verrucaria sp. & 2 & 2 & 4 \\
\hline Rinodina sp. & 1 & 13 & 1 \\
\hline Teloschistes exilis & 1 & 3 & 8 \\
\hline Parmotrema spinibarbe & 1 & 3 & 7 \\
\hline Bulbothrix tabacina & 1 & 3 & 1 \\
\hline Bulbothrix sp. 1 & 1 & 3 & 1 \\
\hline Parmotrema subsumptum & 1 & 1 & 3 \\
\hline Parmotrema cf. epicladum & 9 & & 28 \\
\hline Lecanora concilianda & 7 & & 13 \\
\hline Parmotrema homotomum & 5 & & 10 \\
\hline Hypotrachyna livida & 4 & & 11 \\
\hline Hypotrachyna intercalanda & 4 & & 7 \\
\hline Parmotrema macrocarpum & 4 & & 4 \\
\hline Parmotrema simulans & 4 & & 3 \\
\hline Canoparmelia cf. conlabrosa & 4 & & 2 \\
\hline Hypotrachyna degelii & 2 & & 15 \\
\hline Hypotrachyna pustulifera & 2 & & 10 \\
\hline Heterodermia albicans & 2 & & 4 \\
\hline Parmotrema reticulatum & 1 & & 30 \\
\hline Parmotrema catarinae & 1 & & 4 \\
\hline Canoparmelia carneopruinata & 1 & & 3 \\
\hline Canoparmelia texana & 1 & & 3 \\
\hline Hypotrachyna palmarum & 1 & & 3 \\
\hline Parmotrema pilosum & 1 & & 2 \\
\hline Bulbothrix sp. 2 & 1 & & 1 \\
\hline Cryptothecia striata & 1 & & \\
\hline Graphis assimilis & 27 & 178 & \\
\hline Myelochroa lindmanii & 7 & 11 & \\
\hline Physcia stellaris & 5 & 17 & \\
\hline Micarea sp. & 5 & 7 & \\
\hline Buellia myriocarpa & 4 & 24 & \\
\hline Lecidea canorubella & 4 & 16 & \\
\hline Melaspilea sp. & 3 & 307 & \\
\hline Graphis tachygrapha & 3 & 8 & \\
\hline Graphis anfractuosa & 2 & 4 & \\
\hline
\end{tabular}


Table 7 (continuation)

\begin{tabular}{|c|c|c|c|}
\hline Species & Branches & Twings & Trunks \\
\hline Vainionora sp. & 1 & 31 & \\
\hline Phaeographis lobata & 1 & 14 & \\
\hline Pertusaria ostiolata & 1 & 6 & \\
\hline Physcia poncinsii & 1 & 6 & \\
\hline Parmotrema lobuliferum & 1 & 4 & \\
\hline Ramalina cf. calcarata & 1 & 3 & \\
\hline Canoparmelia cf. scrobicularis & 1 & 1 & \\
\hline Lecanactis sp. & 1 & 1 & \\
\hline
\end{tabular}

There was a change in the community structure related to competition for environmental conditions and specific needs suitable for the establishment of each species. The competition among these species causes a replacement that changes the community structure throughout the tree.

It is clear that the lichen community on D. viscosa varies conditioned to bark roughness that changes as the substrate ages. But this might not be the single factor responsible for the community variation. Luminosity also appears to influence its occurrence. A very detailed approach to microclimate would be necessary in order for a precise clarification on this subject.

\section{Acknowledgements}

The authors thank Dr. Robert Lücking for confirming the crustose species identifications; the Botanical Institute of São Paulo for the opportunity and data support; the Zoobotanical Foundation of Rio Grande do Sul for logistic support; the Forests and Protected Areas Department of the Rio Grande do Sul State Department of the Environment for authorizing this study to be developed in the park area; Natália Koch for making the abstract; Cleodir Mansan, Mariano Pairet and Alessandra B. Lemos for helping during the field work.

\section{Literature cited}

Ahti, T. 2000. Cladoniaceae. Flora Neotropica Monograph 78: 1-362.

Arvidsson, L. 1983. Taxonomical studies in the lichen families Coccocarpiaceae and Pannariaceae. Göteborgs Universitet, Göteborg.
Brodo, I.M. 1973. Substrate ecology. In: V. Ahmadjian \& M.E. Hale Jr. (ed.), The Lichens. Academic Press, New York, pp. 401-436.

Brodo, I.M., Sharnoff, S.D. \& Sharnoff, S. 2001. Lichens of North America. Yale University Press, New Haven.

Canêz, L.S. 2005. A família Parmeliaceae na localidade de Fazenda da Estrela, município de Vacaria, Rio Grande do Sul, Brasil. Dissertação de Mestrado, Instituto de Botânica, São Paulo.

Cáceres, M.E.S., Lücking, R. \& Rambold, G. 2007. Phorophyte specificity and stochasticity as determinants for species composition of corticolous crustose lichen communities in the Atlantic rain forest of northeastern Brazil. Mycological Progress, Tübingen 6: 117-136.

Cáceres, M.E.S., Lücking, R. \& Rambold, G. 2008. Corticolous microlichens in northeastern Brazil: habitat differentiation between coastal Mata Atlântica, Caatinga and Brejos de Altitude. The Bryologist 111: 98-117

Cornelissen, J.H.C. \& Gradstein, S.R. 1990. On the occurrence of bryophytes and macrolichens in different lowland rain forest types at Mabura Hill, Guyana. The Bryology 3: 29-35.

Degelius, G. 1964. Biological studies of the epiphytic vegetation on the twigs of Fraxinus excelsior. Acta Horti Gotoburguensis 27: 12-55.

Delaney, P.J.V. 1965. Fisiografia e geologia de superfície da planície costeira do Rio Grande do Sul. Universidade Federal do Rio Grande do Sul, Porto Alegre.

Dodge, C.W. 1964. Some lichens of tropical Africa. Nova Hedwigia 12: 179-184.

Eliasaro, S. 2001. Estúdio taxonômico y floristico sobre las Parmeliaceae sensu stricto (Ascomycota liquenizados) del segundo Planalto del Estado de Paraná, Brasil. Tesis de Doctor, Universidad de Buenos Aires, Facultad de Ciencias Exactas y Naturales, Buenos Aires.

Eliasaro, S. \& Adler, M. 2000. The species of Canomaculina, Myelochroa, Parmelinella and Parmelinopsis (Parmeliaceae, Lichenezed Ascomycotina) from the "Segundo Planalto" in the State of Paraná, Brazil. Acta Botanica Brasilica 14: 127-139. 
Elix, J.A. 1994. Flora of Austrália. Lichens - Lecanorales 2, Parmeliaceae. Australian Biological Resources Study 55: 5-360.

Fleig, M. 1997. Os gêneros Parmotrema, Rimelia e Rimeliella (Lichenes-Ascomycotina, Parmeliaceae) no Rio Grande do Sul, Brasil. Tese de Doutorado, Universidade de São Paulo, São Paulo.

Fleig, M. 1999. O gênero Pseudocyphellaria (liquens) no Rio Grande do Sul, Brasil. Pesquisas Botânica, Porto Alegre 49: 163-179.

Fortes, A.B. 1959. Geografia física do Rio Grande do Sul. Globo, Porto Alegre.

Galloway, D.J. 1985. Flora of New Zealand - Lichens. P. D. Hasselberg. Government Printer, Wellington.

Galloway, D.J. 2001. Lobariaceae. In: P.M. McCarthy (ed.). Flora of Australia. Volume 58A. Lichens 3. ABRS/ CSIRO, Melbourne, pp. 37-101.

Hale, M.E. 1955. Phytosociology of corticicolous cryptogams in the upland forests of Southern Wisconsin. Ecology 36: 45-63.

Hale, M.E. 1957. Lectures notes Lichenology. West Virginia University, Morgantown.

Hale, M.E. 1976. A monograph of the lichen genus Bulbothryx Hale (Parmeliaceae). Smithsonian Contributions to Botany 32: 1-29.

Hale, M.E. 1983. The Biology of Lichens. 3 ed. Edward Arnold, London.

Hawksworth, D.L. 1975. Lichens - new introduction, matter and supplementary. The Richmond Publishing, Cambridge.

Hawksworth, D.L. \& Hill, D.J. 1984. The LichenForming Fungi. Blackie, New York.

Jesberger, J.A. \& Sheard, J.W. 1973. A quantitative study and multivariate analysis of corticolous lichen communities in the Southern boreal forest of Saskatchewan. Canadian Journal of Botany 51: 185-201.

Jørgensen, P.M. \& James, P.W. 1983. Studies on some Leptogium species western Europe. Lichenologist 33: 483-485.

Jungbluth, P. 2006. A família Parmeliaceae (fungos liquenizados) em fragmentos de cerrados do Estado de São Paulo. Dissertação de Mestrado, Instituto de Botânica, São Paulo.

Käffer, M.I., Ganade, G. \& Marcelli, M.P. 2007. Interação entre liquens e forófitos em quarto ambientes na FLONA de São Francisco de Paula. Revista Brasileira de Biociências 5: 216-218.

Käffer, M.I., Ganade, G. \& Marcelli, M.P. 2009. Lichen diversity and composition in Araucaria forest and tree monocultures in southern Brazil. Biodiversity and Conservation 18: 3543-356.

Käffer, M.I., Marcelli, M.P. \& Ganade, G. 2010. Distribution and composition of the lichenized mycota in a landscape mosaic of southern Brazil. Acta Botanica Brasilica 24: 790-802.
Komposch, H. \& Hafellner, J. 2000. Diversity and vertical distribution of lichens in a Venezuelan tropical lowland rain forest. Selbyana 21: 11-24.

Komposch, H. \& Hafellner, J. 2003. Species composition of lichen dominated corticolous communities: a lowland rain Forest canopy compared to na adjacent shrubland in Venezuela. Bibliotheca Lichenologica 86: 351-367.

Marcelli, M.P. 1987. Ecologia dos liquens dos manguezais da região Sul-Sudeste do Brasil, com especial atenção ao de Itanhaém (SP). Tese de Doutorado, Universidade de São Paulo, São Paulo.

Marcelli, M.P. 1990. Liquens de restingas e manguezais da Ilha do Cardoso. In: Anais do $2^{\circ}$ Simpósio de Ecossistemas da Costa Sul e Sudeste Brasileira, Águas de Lindóia, v. 3, pp. 382-392.

Marcelli, M.P. 1991. Aspects of the foliose lichen flora of the southern-central coast of São Paulo State, Brazil. In: D.J. Galloway (ed.). Tropical lichens: Their systematics, conservation, and ecology. Systematics Association Special. Clarendon Press, Oxford, pp. 151-170.

Marcelli, M.P. 1992. Ecologia liquênica nos manguezais do Sul-Sudeste brasileiro. Bibliotheca Lichenologica 47: $1-310$.

Marcelli, M.P. 1995. Habitat selection of epiphytic lichens on Rhizophora mangle in the mangroves of the Itanhaém river, São Paulo, Brazil. In: F.J.A. Daniels, M. Schultz \& J. Peine (eds.) Flechten Follmann, Contributions to Lichenology in Honour of Gerhard Follmann. Geobotanical and Phytotaxonomical Study Group, Botanical Institute, University of Cologne, Cologne, pp. 533-541.

Marcelli, M.P. 1996. Biodiversity assessment in lichenized fungi: the necessary naive roll makers. In: C.E. Bicudo \& N.A. Menezes (eds.). Biodiversity in Brazil: A first approach. Conselho Nacional do Desenvolvimento Científico e Tecnológico, São Paulo, pp. 93-107.

Marcelli, M.P. \& Ahti, T. 1998. Recollecting Edvard August Vainio. Cetesb, São Paulo.

Moberg, R. 1989. The lichen genus Physcia in Central and South America. Nordic Journal Botany 10: 319-342.

Nash III, T.H. 1996. Photosynthesis, respiration, productivity and growth. In: T.H. Nash III (ed.). Lichen Biology. Cabridge University Press, Cambridge, pp. 88-120.

Pearson, L.C. 1969. Influence of temperature and humidity on distribution of lichens in a Minnesota bog. Ecology 50: $740-746$.

Pedersen, I. 1980. Epiphytic lichen vegetation in an old oak wood, Kaas Skov. Botany Tidsskrift 75: 105-120.

Reitz, R. 1980. Sapindáceas. In: R. Reitz (ed.). Flora Ilustrada Catarinense. Herbário Barbosa Rodrigues, Itajaí, pp. 1-156.

Ribeiro, C.H. 1998. A família Parmeliaceae (Ascomycota liquenizados) em regiões montanhosas dos Estados de Minas Gerais, Rio de Janeiro e São Paulo. Dissertação de Mestrado, Universidade de São Paulo, São Paulo. 
Ruchty, A., Rosso, A.L. \& McCune, B. 2001. Changes in epiphyte communities as the shrub, Acer circinatum develops and ages. The Bryologist 104: 274-281.

Schmidt, J., Kricke, R. \& Feige, G.B. 2001. A measurements of bark $\mathrm{pH}$ with a modified flathead electrode. The Lichenologist 33: 456-460.

Scutari, N.C. 1992. Estúdios sobre Pyxinaceae foliosas (Lecanorales, Ascomycotina) de la Argentina, IV: claves de los generos y las especies de la Provincia de Buenos Aires. Boletín Sociedad Argentina de Botanica 28: 169-173.

Scutari, N.C. 1995. Los macrolíquenes de Buenos Aires, I: Dirinaria, Heterodermia e Hyperphyscia (Physciaceae, Ascomycotina). Darwiniana 33: 149-176.

Sérusiaux, E. 1983. New data on the lichen genus Punctelia (Parmeliaceae). Nordic Journal of Botany 3: 517-520.

Sipman, H.J.M. 2002. Mason Hale's key to Parmotrema, revised edition: key to wide-lobed parmelioid species occuring in Tropical America (genera Canomaculina, Parmotrema, Rimelia, Rimeliella). http://www.bgbm.org/sipman/keys/Neoparmo.htm (access in: 18.09.2006).
Swinscow, T.D.V. \& Krog, H. 1998. Macrolichens of East Africa. British Museum of Natural History, London.

Teixeira, M.B., Coura-Neto, A.B., Pastore, U. \& Rangel Filho, A.L.R. 1986. Vegetação: As regiões fitoecológicas, sua natureza e seus recursos econômicos - Estudo fitogeográfico. In: Levantamento de Recursos Naturais. Instituto Brasileiro de Geografia e Estatística, Rio de Janeiro 33: 541-620.

Ward, J.H. 1963. Hierarchical grouping to optimise an objective function. Journal of the American Statistical Association 58: 236-244.

Werth, S. 2001. Key factors for epiphytic macrolichen vegetation in deciduous forests of Troms country, northern Norway: human impact, substrate climate or spatial variation? Doctoral Thesis Departament of Biology, Faculty of Science, University of Tronsø, Tronsø.

Wolf, J.H.D. 1993. Diversity patterns and biomass of epiphytic bryophytes and lichens along an altitudinal gradient in the northern Andes. Annals of the Missouri Botanical Garden 80: 928- 960. 